DOI: $10.21105 /$ joss. 02868

\title{
ivadomed: A Medical Imaging Deep Learning Toolbox
}

\author{
Charley Gros ${ }^{1,2}$, Andreanne Lemay ${ }^{1,2}$, Olivier Vincent ${ }^{1,2}$, Lucas \\ Rouhier $^{1}$, Marie-Helene Bourget ${ }^{1}$, Anthime Bucquet ${ }^{1}$, Joseph Paul \\ Cohen $^{2,3}$, and Julien Cohen-Adad ${ }^{1,2,4}$
}

1 NeuroPoly Lab, Institute of Biomedical Engineering, Polytechnique Montreal, Montreal, Canada 2 Mila, Quebec Al Institute, Montreal, QC, Canada 3 AIMI, Stanford University, Stanford, CA, USA 4 Functional Neuroimaging Unit, CRIUGM, Université de Montréal, Montreal, QC, Canada

\section{Software}

- Review ¿

- Repository ¿

- Archive [

\section{Summary}

Editor: Christopher R. Madan ¿ Reviewers:

- @NMontanaBrown

- Qjustusschock

- Qlbugnon

Submitted: 11 November 2020 Published: 12 February 2021

\section{License}

Authors of papers retain copyright and release the work under a Creative Commons Attribution 4.0 International License (CC BY 4.0).

ivadomed is an open-source Python package for designing, end-to-end training, and evaluating deep learning models applied to medical imaging data. The package includes APIs, commandline tools, documentation, and tutorials. ivadomed also includes pre-trained models such as spinal tumor segmentation and vertebral labeling. Original features of ivadomed include a data loader that can parse image and subject metadata for custom data splitting or extra information during training and evaluation. Any dataset following the Brain Imaging Data Structure (BIDS) convention will be compatible with ivadomed. Beyond the traditional deep learning methods, ivadomed features cutting-edge architectures, such as FiLM (Perez et al., 2017) and HeMis (Havaei et al., 2016), as well as various uncertainty estimation methods (aleatoric and epistemic), and losses adapted to imbalanced classes and non-binary predictions. Example applications of ivadomed include MRI object detection, segmentation, and labeling of anatomical and pathological structures. ivadomed's main project page is available at https://ivadomed.org.

\section{Statement of need}

Deep learning applied to medical imaging presents many challenges: datasets are often not publicly-available, ground truth labels are difficult to produce, and needs are usually tailored to particular datasets and tasks (Kim et al., 2019). There already exists a few deep learning frameworks for medical imaging, but each has their pros \& cons. ivadomed notably addresses unmet needs in terms of data management, readily-available uncertainty outputs, missing modalities (in case of multi-channel training) and model comparison, to only name few of the original features.

Another challenge of medical imaging is the heterogeneity of the data across hospitals (e.g., contrast, resolution), making it difficult to create models that can generalize well. Recent cutting-edge methods address this problem, such as FiLM (Perez et al., 2017) and HeMis (Havaei et al., 2016), however they are usually not implemented within a comprehensive framework. In addition to providing these architectures, ivadomed features losses adapted to imbalanced classes and non-binary predictions. 


\section{ivad:med}

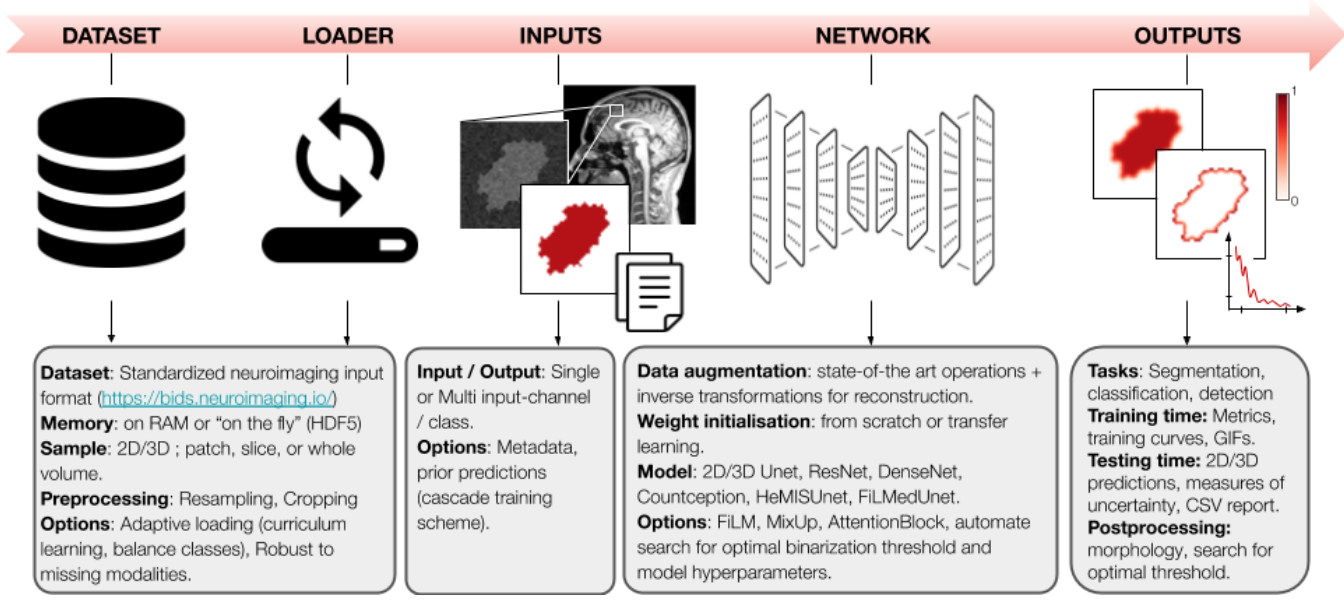

Figure 1: Overview of ivadomed main features.

\section{Loader}

Standard data structure: In machine learning, lots of time is spent curating the data (renaming, filtering per feature) before entering the training pipeline (Willemink et al., 2020). ivadomed features an advanced data loader compatible with a standardized data structure in neuroimaging: the Brain Imaging Data Structure (BIDS) (Gorgolewski et al., 2016). Thus, any dataset following the BIDS convention can readily be used by ivadomed. BIDS convention is originally designed around MRI data and accepts NIfTI file formats, but the BIDS community is actively expanding its specifications to other modalities (CT, MEG/EEG, microscopy), which ivadomed will then be able to accommodate.

Access to metadata: One benefit of the BIDS convention is that each image file is associated with a JSON file containing metadata. ivadomed's loader can parse image metadata (e.g., acquisition parameters, image contrast, resolution) and subject metadata (e.g., pathology, age, sex) for custom data splitting or extra information during training and evaluation. It is possible to modulate specific layers of a convolutional neural network using metadata information to tailor it towards a particular data domain or to enable experiments with architectures such as FiLM (Perez et al., 2017). Metadata could also be useful to mitigate class imbalance via data balancing techniques.

\section{Training}

Architectures: ivadomed includes all the necessary components for training segmentation models from start to finish, including data augmentation transformations and transfer learning. Available architectures include: 2D U-Net (Ronneberger et al., 2015), 3D U-Net (Isensee et al., 2017), ResNet (He et al., 2016), DenseNet (Huang et al., 2017), Count-ception (Cohen et al., 2017), and HeMIS U-Net. These models can easily be enriched via attention blocks (Oktay et al., 2018) or FiLM layers (which modulate U-Net features using metadata).

Losses and class imbalance: Popular losses are available: Dice coefficient (Milletari et al., 2016), cross-entropy, and L2 norm, including some adapted to medical imaging challenges, such as the adaptive wing loss (X. Wang et al., 2019) for soft labels and the focal Dice loss (Wong et al., 2018) for class imbalance. Useful to alleviate overfitting, mixup (Zhang et al., 


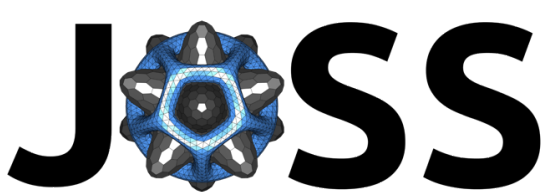

The Journal of Open Source Software

2017) was modified to handle segmentation tasks. To mitigate class imbalance, ivadomed supports cascaded architectures. With a single inference, it is possible to narrow down the region of interest via object detection and then segment a specific structure, as illustrated in Figure 2.

Getting started: It can be overwhelming to get started and choose across all the available models, losses, and parameters. ivadomed's repository includes the script ivadomed_automa te_training to configure and launch multiple trainings across GPUs. In case of interruption during training, all parameters are saved after each epoch so that training can be resumed at any time.

\section{Evaluation}

Uncertainty: Aleatoric (G. Wang et al., 2019) and/or epistemic (Nair et al., 2020) uncertainties can be computed voxel-wise and/or object-wise (Roy et al., 2018). Multiple metrics are available, including entropy and coefficient of variation.

Post-processing: Predictions can be conveniently filtered using popular methods, e.g., fill holes, remove small objects, threshold using uncertainty. It is also possible to compute metrics for specific object sizes (e.g., small vs. large lesions). ivadomed has a module to find the optimal threshold value on the soft output prediction, via a grid-search finding applied to evaluation metrics or ROC curve.

Visualize performance: Convenient visualization tools are available for model design and optimization: GIF animations across training epochs, visual quality control of data augmentation, training curve plots, integration of the TensorBoard module, and output images with true/false positive labels. See this example tutorial.

\section{Usage}

Past and ongoing research projects using ivadomed are listed here. The figure below illustrates a cascaded architecture for segmenting spinal tumors on MRI data (Lemay et al., 2020).

Step 1: Localize spinal cord

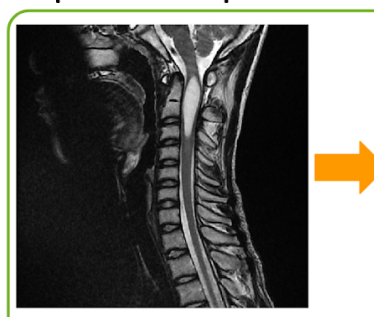

Input image

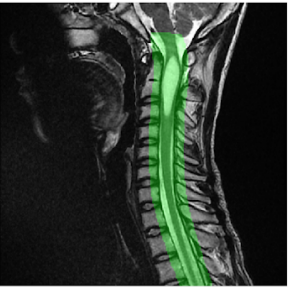

Spinal cord localization

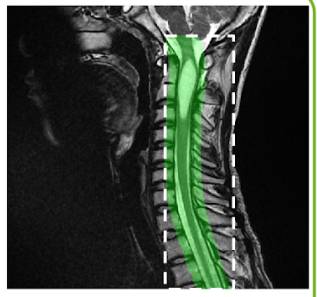

Cropping
Step 2: Segment tumor

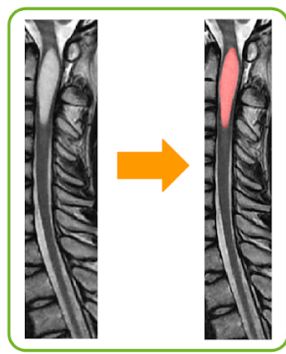

Figure 2: Fully automatic spinal cord tumor segmentation framework..

\section{Acknowledgements}

The authors thank Ainsleigh Hill, Giselle Martel, Alexandru Jora, Nick Guenther, Joshua Newton, Christian Perone, Konstantinos Nasiotis, Valentine Louis-Lucas, Benoît Sauty-DeChalon, Alexandru Foias and Leander Van Eekelen for their useful contributions, and Guillaume Dumas for proof-reading the manuscript. Funded by IVADO, the Canada Research Chair in 
Quantitative Magnetic Resonance Imaging [950-230815, 950-233166], CIHR [FDN-143263], CFI [32454, 34824], FRQS [28826], NSERC [RGPIN-2019-07244], FRQNT [2020-RS4-265502 UNIQUE] and TransMedTech. C.G. has a fellowship from IVADO [EX-2018-4], A.L. has a fellowship from NSERC, FRQNT and UNIQUE, O.V. has a fellowship from NSERC, FRQNT and UNIQUE, M.H.B. has a fellowship from IVADO and FRQNT.

\section{References}

Cohen, J. P., Boucher, G., Glastonbury, C. A., Lo, H. Z., \& Bengio, Y. (2017). Countception: Counting by Fully Convolutional Redundant Counting. International Conference on Computer Vision Workshop on Biolmage Computing. https://doi.org/10.1109/iccvw. 2017.9

Gorgolewski, K. J., Auer, T., Calhoun, V. D., Craddock, R. C., Das, S., Duff, E. P., Flandin, G., Ghosh, S. S., Glatard, T., Halchenko, Y. O., Handwerker, D. A., Hanke, M., Keator, D., Li, X., Michael, Z., Maumet, C., Nichols, B. N., Nichols, T. E., Pellman, J., ... Poldrack, R. A. (2016). The brain imaging data structure, a format for organizing and describing outputs of neuroimaging experiments. Scientific Data, 3(1), 160044. https: //doi.org/10.1038/sdata.2016.44

Havaei, M., Guizard, N., Chapados, N., \& Bengio, Y. (2016). HeMIS: Hetero-modal image segmentation. arXiv Preprint arXiv:1607.05194. https://doi.org/10.1007/ 978-3-319-46723-8_54

He, K., Zhang, X., Ren, S., \& Sun, J. (2016). Deep Residual Learning for Image Recognition. Conference on Computer Vision and Pattern Recognition, 770-778. https://doi.org/10. 1109/CVPR.2016.90

Huang, G., Liu, Z., Maaten, L. van der, \& Weinberger, K. Q. (2017). Densely Connected Convolutional Networks. Computer Vision and Pattern Recognition. https://arxiv.org/ abs/1608.06993

Isensee, F., Kickingereder, P., Wick, W., Bendszus, M., \& Maier-Hein, K. H. (2017). Brain tumor segmentation and radiomics survival prediction: Contribution to the BRATS 2017 challenge. International MICCAI Brainlesion Workshop, 287-297.

Kim, M., Yun, J., Cho, Y., Shin, K., Jang, R., Bae, H., \& Kim, N. (2019). Deep Learning in Medical Imaging. Neurospine, 16(4), 657-668. https://doi.org/10.14245/ns.1938396.198

Lemay, A., Gros, C., Zhuo, Z., Zhang, J., Duan, Y., Paperi, S., Cohen-Adad, J., \& Liu, Y. (2020, July). Fully Automatic Spinal Cord Tumor Segmentation on MRI with Deep Learning. Organization for Human Brain Mapping.

Milletari, F., Navab, N., \& Ahmadi, S.-A. (2016). V-net: Fully convolutional neural networks for volumetric medical image segmentation. 2016 Fourth International Conference on 3d Vision (3DV), 565-571. https://doi.org/10.1109/3dv.2016.79

Nair, T., Precup, D., Arnold, D. L., \& Arbel, T. (2020). Exploring uncertainty measures in deep networks for multiple sclerosis lesion detection and segmentation. Medical Image Analysis, 59, 101557. https://doi.org/10.1016/j.media.2019.101557

Oktay, O., Schlemper, J., Folgoc, L. L., Lee, M., Heinrich, M., Misawa, K., Mori, K., McDonagh, S., Hammerla, N. Y., Kainz, B., \& others. (2018). Attention u-net: Learning where to look for the pancreas. arXiv Preprint arXiv:1804.03999.

Perez, E., Strub, F., De Vries, H., Dumoulin, V., \& Courville, A. (2017). Film: Visual reasoning with a general conditioning layer. arXiv Preprint arXiv:1709.07871. 
Ronneberger, O., Fischer, P., \& Brox, T. (2015, May). U-Net: Convolutional Networks for Biomedical Image Segmentation. Medical Image Computing and Computer Assisted Intervention. https://arxiv.org/abs/1505.04597

Roy, A. G., Conjeti, S., Navab, N., \& Wachinger, C. (2018). QuickNAT: A Fully Convolutional Network for Quick and Accurate Segmentation of Neuroanatomy. arXiv:1801.04161 [cs]. https://doi.org/10.1016/j.neuroimage.2018.11.042

Wang, G., Li, W., Aertsen, M., Deprest, J., Ourselin, S., \& Vercauteren, T. (2019). Aleatoric uncertainty estimation with test-time augmentation for medical image segmentation with convolutional neural networks. Neurocomputing, 338, 34-45. https://doi.org/10.1016/j. neucom.2019.01.103

Wang, X., Bo, L., \& Fuxin, L. (2019). Adaptive wing loss for robust face alignment via heatmap regression. Proceedings of the IEEE International Conference on Computer Vision, 6971-6981. https://doi.org/10.1109/iccv.2019.00707

Willemink, M. J., Koszek, W. A., Hardell, C., Wu, J., Fleischmann, D., Harvey, H., Folio, L. R., Summers, R. M., Rubin, D. L., \& Lungren, M. P. (2020). Preparing medical imaging data for machine learning. Radiology, 295(1), 4-15.

Wong, K. C., Moradi, M., Tang, H., \& Syeda-Mahmood, T. (2018). 3D segmentation with exponential logarithmic loss for highly unbalanced object sizes. International Conference on Medical Image Computing and Computer-Assisted Intervention, 612-619. https://doi. org/10.1007/978-3-030-00931-1_70

Zhang, H., Cisse, M., Dauphin, Y. N., \& Lopez-Paz, D. (2017). Mixup: Beyond empirical risk minimization. arXiv Preprint arXiv:1710.09412. 